\title{
Prática segura para partos em hospital universitário
}

Safe practice for childbirth in a university hospital

Práctica segura para partos en un hospital universitario

\section{Maxuel Cruz dos Santos ${ }^{\mathrm{I}}$, Vitória Sandri Pedroni' ${ }^{\mathrm{II}}$, Franciela Delazeri Carlotto ${ }^{\mathrm{III}}$, Silvana Cruz da Silva $^{\mathrm{IV}}$, Helga Geremias Gouveiav ${ }^{\mathrm{V}}$, Letícia Becker Vieira ${ }^{\mathrm{VI}}$}

\begin{abstract}
Resumo: Objetivo: identificar a aplicação das práticas essenciais da Lista de Verificação para Partos Seguros (LVPS) da Organização Mundial da Saúde realizadas em Hospital Universitário. Método: estudo transversal descritivo, realizado no período de janeiro a outubro de 2018, com 51 profissionais que assistem o trabalho de parto, parto e puerpério. Para identificar as práticas aplicadas, utilizou-se um questionário semiestruturado e análise estatística. Resultados: práticas relacionadas ao uso de fármacos, disponibilidade de recursos materiais, identificação de sangramento anormal, contato pele à pele, amamentação e planejamento reprodutivo estão conforme o preconizado. Contudo, não houve padronização quanto às avaliações no partograma e às orientações às mulheres e acompanhantes sobre os sinais de agravamento. Conclusões: a LVPS apresenta-se como uma ferramenta inovadora na assistência obstétrica. Oferece oportunidade de melhorias e qualificação dos cuidados, padronizando condutas essenciais, como as orientações sobre os sinais clínicos e registro no partograma, favorecendo a segurança do binômio mãe-bebê.
\end{abstract}

Descritores: Lista de checagem; Segurança do paciente; Cuidados de enfermagem; Trabalho de parto; Obstetrícia

Abstract: Objective: to identify the application of the essential practices of the World Health Organization Checklist for Safe Births (CLSB) performed in a University Hospital. Method: a descriptive cross-sectional study, carried out from January to October 2018, with 51 professionals who assist in labor, delivery and puerperium. To identify the applied practices, a semi-structured questionnaire and statistical analysis were used. Results: practices

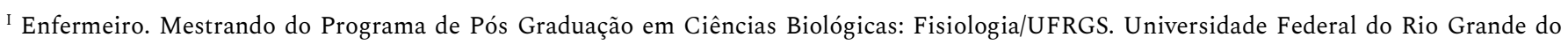
Sul. Porto Alegre, Rio Grande do Sul, Brasil. maxuelcsantos@gmail.com. https://orcid.org/0000-0001-6750-2265

II Enfermeira. Graduação em Enfermagem. Universidade Federal do Rio Grande do Sul. Porto Alegre, Rio Grande do Sul, Brasil. vispedroni@gmail.com. https://orcid.org/0000-0002-2936-1497

III Acadêmica de Enfermagem. Universidade Federal do Rio Grande do Sul. Porto Alegre, Rio Grande do Sul, Brasil. francielacarlotto@gmail.com; https://orcid.org/0000-0003-4187-0603

IV Enfermeira. Doutora em Enfermagem pelo Programa de Pós-Graduação em Enfermagem da Universidade Federal do Rio Grande do Sul/ UFRGS. Professora de Enfermagem da Universidade Franciscana. Santa Maria, Rio Grande do Sul, Brasil. silvanacruzufsm@yahoo.com.br https://orcid.org/0000-0002-4563-3704

V Enfermeira. Doutora em Ciências da Saúde na área de Saúde Materna e Perinatal. Professora Associada Universidade Federal do Rio Grande do Sul. Porto Alegre, Rio Grande do Sul, Brasil. helgagouveia@hotmail.com https://orcid.org/0000-0003-4980-4141

VI Enfermeira. Doutora em Enfermagem. Professora Adjunta Universidade Federal do Rio Grande do Sul. Porto Alegre, RS, Brasil. lebvieira@hotmail.com https://orcid.org/0000-0001-5850-7814
} 
related to the use of drugs, availability of material resources, identification of abnormal bleeding, skin-to-skin contact, breastfeeding and reproductive planning are as recommended. However, there was no standardization regarding the evaluations in the partogram and the orientations to women and companions about the signs of worsening. Conclusions: the CLSB presents itself as an innovative tool in obstetric care. It offers opportunities for improvement and qualification of care, standardizing essential conducts, such as guidelines on clinical signs and registration in the partogram, favoring the safety of the mother-baby binomial.

Descriptors: Checklist; Patient safety; Nursing care; Labor; Obstetrics

Resumen: Objetivo: identificar la aplicación de las prácticas esenciales de la Lista de Verificación para Partos Seguros (LVPS) de la Organización Mundial de la Salud que se realizan en un Hospital Universitario. Método: estudio transversal y descriptivo realizado entre los meses de enero y octubre de 2018 con 51 profesionales que asisten en el trabajo de parto, en el parto y durante el puerperio. Para identificar las prácticas aplicadas se utilizaron un cuestionario semiestructurado y análisis estadístico. Resultados: las prácticas relacionadas con el uso de fármacos, la disponibilidad de recursos materiales, la identificación de sangrado anormal, el contacto piel a piel, el amamantamiento y la planificación reproductiva se condicen con las recomendaciones. Pese a todo, no hubo estandarización en cuanto a las evaluaciones en el partograma y a las orientaciones ofrecidas a las mujeres y a sus acompañantes sobre las señales de agravamiento del cuadro. Conclusiones: la LVPS se presenta como una herramienta innovadora en la asistencia obstétrica. Ofrece la oportunidad de mejorar y calificar los cuidados, estandarizando conductas esenciales como ser las orientaciones sobre las señales clínicas y el registro en el partograma, favoreciendo así la seguridad del binomio madre-bebé.

Descriptores: Lista de verificación; Seguridad del paciente; Cuidados de enfermería; Trabajo de parto; Obstetricia

\section{Introdução}

A segurança do paciente é um importante pilar dos processos relacionados à qualificação da assistência nas instituições de saúde. O Brasil é um dos países que compõem a Aliança Mundial estabelecida pela Organização Mundial de Saúde (OMS), em 2004, cujo principal propósito é instituir medidas que aumentem a qualidade desses serviços e, por conseguinte, a segurança do paciente. ${ }^{1}$

No que se refere à atenção obstétrica, a qualificação da assistência associada à segurança do paciente são bases para redução das taxas de mortalidade materna e infantil. ${ }^{1}$ Destaca-se a magnitude dos casos, uma vez que 830 mulheres morrem por dia, no mundo, por problemas relacionados à gravidez e ao parto. Estima-se que melhores cuidados antes e após o parto evitariam mortes maternas e infantis, ${ }^{2}$ podendo haver uma redução de 1,49 milhões desses óbitos por ano em todo o mundo. ${ }^{3}$ 
3 | Santos MC, Pedroni VS, Carlotto FD, Silva SC, Gouveia HG, Vieira LB

Indica-se para isso, que a utilização de tecnologias na saúde possa qualificar a assistência materna e perinatal, visando melhorias contínuas dos processos de cuidado e consequentemente a maior segurança ao binômio mãe-bebê. ${ }^{1}$ Dentre as tecnologias, destaca-se a criação de protocolos de segurança, recomendados pela OMS, a qual, em 2017, lançou o guia para implementação da Lista de Verificação para Partos Seguros (LVPS). O intuito é a redução dos eventos adversos evitáveis na assistência materna e perinatal, por meio de práticas simples e efetivas. ${ }^{4}$

O desenvolvimento e a utilização de listas de verificação para a área da saúde são ferramentas práticas e fáceis de serem utilizadas pelos profissionais de saúde. A LVPS é composta por 29 práticas recomendadas mundialmente, que sintetizam as melhores e mais atuais evidências científicas, o que favorece à redução das principais causas de mortes maternas e de recém-nascidos, tais como: hemorragia, infecções, pré-eclâmpsia e eclâmpsia.,5-6 Os itens foram agrupados em quatro seções, caracterizando as etapas que o profissional deve realizar a verificação, sendo estas, no momento da admissão, antes da expulsão (ou antes da cesariana), logo após o parto (no espaço de uma hora) e antes da alta. ${ }^{4}$

Inicialmente, a LVPS foi testada em nove países da África e Ásia, em 2010, e posteriormente revista no estado de Karnataka, na Índia. Estes estudos evidenciaram, após a implementação da lista, o aumento de 10 para 25, das 29 práticas essenciais (seguras) de assistência ao parto pelos profissionais. ${ }^{7-9}$ Pesquisa no sul da África, avaliando a LVPS, destaca a redução da mortalidade perinatal de 22 para 13,8 por 1000 nascidos vivos. ${ }^{5}$

$\mathrm{Na}$ realidade brasileira, estudo adaptou e validou a LVPS, ressaltando sua importância, visto que contempla boas práticas de segurança do paciente e avalia a mulher e o recém-nascido desde sua admissão até a alta hospitalar. ${ }^{10} \mathrm{O}$ guia de implementação da LVPS prevê adaptações para as diferentes realidades, de acordo com o local e especificidades geográficas. ${ }^{11}$ Prevê ainda a participação dos profissionais de saúde, principalmente enfermeiros e médicos, para sua 
efetiva utilização, ${ }^{4}$ visto que a adoção de uma nova rotina de segurança do paciente requer envolvimentos e formação profissional. ${ }^{12}$

Considerando-se que a LVPS corresponde a um documento recente, que carece de mais estudos no contexto brasileiro para caracterizar a sua implantação, adesão pelos profissionais e repercussão na qualidade da assistência, tem-se como questão de pesquisa: quais práticas essenciais preconizadas pela LVPS são aplicadas pelos enfermeiros e médicos em um Hospital Universitário no Sul do Brasil? Dessa maneira, o estudo tem como objetivo identificar a aplicação das práticas essenciais da LVPS da OMS realizadas em Hospital Universitário.

\section{Método}

Trata-se de um estudo transversal descritivo de abordagem quantitativa, realizado no Serviço Materno Infantil de um Hospital Universitário, que atende mulheres gestantes de risco habitual, sendo também referência para assistência à gestação de alto risco para todo o Estado do Rio Grande do Sul. Tem uma média de 50 atendimentos diários e cerca de 10 partos, os quais totalizaram, em 2017, 3.415 partos, sendo a taxa de cesárea de 36,8\%.

O Serviço Materno é composto por Unidade de Centro Obstétrico (UCO) e Unidade de Internação Obstétrica (UIO). Na UCO, são sete salas de pré-parto, três leitos de observação, sete leitos de recuperação e cuidados com o recém-nascido, duas salas de parto e duas salas de cesárea. A UIO é composta por 45 leitos, sendo seis destinados a gestantes de alto risco, atendendo pacientes provenientes do Sistema Único de Saúde (SUS), convênio e particular.

A população do estudo foi de 72 profissionais; 30 enfermeiras, 20 médicas obstetras e 22 neonatologistas, que atuam no trabalho de parto, parto e puerpério, desde a admissão até a alta hospitalar. A amostra foi por conveniência. Foram incluídos todos os profissionais com mais de seis meses de atuação nas unidades, seis profissionais foram excluídos por estarem em férias, licença saúde ou maternidade, três por não estarem atuando na área, no período de coleta de 
5 | Santos MC, Pedroni VS, Carlotto FD, Silva SC, Gouveia HG, Vieira LB

dados e 12 profissionais recusaram-se a participar. Assim, participaram da pesquisa 51 profissionais. Dentre eles, 26 enfermeiras, sendo 16 enfermeiras obstetras que atuavam na UCO e 10 enfermeiras que atuavam na UIO; e 25 médicas, sendo nove obstetras e 16 neonatologistas.

A coleta de dados ocorreu no período de janeiro a outubro de 2018, por uma equipe de pesquisa capacitada. Utilizou-se um questionário semiestruturado, aplicado aos profissionais fora do seu horário de trabalho em dia escolhido por eles. O instrumento foi composto pelos quatro momentos de pausa previstos pela LVPS e as perguntas eram direcionadas às práticas desenvolvidas no cuidado do binômio mãe-bebê desde a admissão até a alta hospitalar. Ou seja, cada profissional respondeu às questões relativas aos cuidados, conforme sua competência.

No momento da admissão, as perguntas eram relativas à verificação da necessidade de transferência da mãe, início do partograma, necessidade de medicações, disponibilidade de material para lavar as mãos e luvas em cada exame vaginal, a presença do acompanhante e orientações para solicitar auxílio. No período antes da expulsão (ou antes da cesariana), os questionamentos eram relativos à verificação da necessidade de medicações, a confirmação do material necessário para realização do parto e sobre a identificação do profissional responsável pelo procedimento. No terceiro momento, logo após o parto (no espaço de uma hora), as questões eram relacionadas à verificação de sangramento anormal, à conduta profissional, ao uso de medicações, à necessidade de transferência do recém-nascido, às medicações ao recémnascido, aos cuidados especiais, à amamentação, ao contato pele à pele, e sobre orientações a solicitar auxílio em sinais de perigo. No período antes da alta, indagava-se sobre a verificação de permanência na unidade por 24 horas, o uso de medicações, a presença de sangramento, a necessidade de antibióticos ao recém-nascido, os motivos de adiamento da alta, as dificuldades em amamentar, as orientações sobre o planejamento familiar, a marcação de consultas de seguimento e sobre as orientações a solicitar auxílio após a alta hospitalar. 
Para organização e análise dos dados, utilizou-se o software Statistical Package for the Social Sciences (SPSS) versão 20.0. Foi realizada dupla digitação e os dados foram analisados quantitativamente, conforme frequências absolutas e relativas. O estudo está vinculado ao projeto matricial intitulado: Processo de implantação do checklist nascimento seguro: ciclo de melhoria da qualidade e a segurança do paciente na obstetrícia, que foi aprovado pelo Comitê de Ética em Pesquisa do Hospital Universitário sob o parecer nº 2.230.927, na data de 21 de agosto de 2017, estando de acordo com a Resolução 466/2012. Foram expostos os objetivos da pesquisa e os participantes que aceitaram participar assinaram o Termo de Consentimento Livre e Esclarecido.

\section{Resultados}

Quanto à caracterização dos participantes, observou-se que $48(94,1 \%)$ eram do sexo feminino, três $(5,9 \%)$ do sexo masculino e que $51(100 \%)$ se autodeclararam brancos. A faixa etária estava entre 29 e 67 anos, tendo como média 43,27 anos. A média do tempo de formação foi de 17,2 anos, com média de atuação na UCO/UIO de 10,59 anos. Em relação à formação complementar lato senso, $37(72,5 \%)$ possuem especialização, sendo em enfermagem obstétrica, obstetrícia e ginecologia, neonatologia, saúde pública, auditoria, imunizações e urgência e emergência. Com relação à formação stricto senso, 18 (35,3\%) têm mestrado e seis $(11,8 \%)$, doutorado.

As ações desenvolvidas (ou não) pelos profissionais são apresentadas em tabelas, de acordo com os momentos preconizados pela LVPS e profissionais envolvidos no cuidado em cada item. Na Tabela 1, estão apresentadas as práticas desenvolvidas por 25 profissionais (nove médicos obstetras e 16 enfermeiras), na UCO, nos três primeiros momentos. Dentre essas, identificou-se que a verificação de alergias, início do partograma na fase ativa, disponibilidade de materiais, indicação para uso de medicações, identificação do profissional responsável, encorajamento da presença do acompanhante, administração de ocitocina e cuidados com 
sangramentos são práticas realizadas. Ao contrário, práticas como a periodicidade de registros no partograma não são realizadas conforme preconizado pela LVPS.

Tabela 1 - Itens da LVPS realizados por médicos obstetras e enfermeiras na UCO. Porto Alegre - RS, 2019.

\begin{tabular}{|c|c|c|c|}
\hline \multirow{2}{*}{$\begin{array}{l}\text { Variável } \\
\text { Momento da admissão }\end{array}$} & \multicolumn{2}{|c|}{$\mathbf{n}=\mathbf{2 5}$} & \multirow[t]{2}{*}{$\%$} \\
\hline & & & \\
\hline 1. Verifica se há necessidade de transferência & 18 & $\operatorname{Sim}$ & 72 \\
\hline 2. Verifica se a gestante tem alergia & 24 & $\operatorname{Sim}$ & 96 \\
\hline 3. Indicação para início do Partogama & 24 & Fase ativa & 96 \\
\hline Periodicidade da avaliação de contrações & 12 & $30 \mathrm{~min}$ & 48 \\
\hline Periodicidade da avaliação dos BCFs & 14 & $30 \mathrm{~min}$ & 56 \\
\hline Periodicidade da avaliação da FC & 20 & 6 horas & 80 \\
\hline Periodicidade da avaliação da Tax & 19 & 6 horas & 76 \\
\hline Periodicidade da avaliação da PA & 19 & 6 horas & 76 \\
\hline \multirow[t]{3}{*}{ 4. Indicação para administração de antibiótico à gestante } & 23 & Infecção & 92 \\
\hline & 15 & Bolsa Rota & 60 \\
\hline & 13 & Profilaxia & 52 \\
\hline 5. Indicação para administração de sulfato de magnésio à & 24 & HA Gestacional & 96 \\
\hline gestante & 16 & Prematuridade & 64 \\
\hline 6. Indicação para administração de Anti-hipertensivos à & 22 & Crise Hipertensiva & 88 \\
\hline \multirow[t]{2}{*}{ gestante } & 13 & HA Crônica & 52 \\
\hline & 07 & HA Gestacional & 28 \\
\hline $\begin{array}{l}\text { 7. Disponibilidade de materiais para lavar as mãos e luvas para } \\
\text { cada exame vaginal }\end{array}$ & 25 & $\operatorname{Sim}$ & 100 \\
\hline 8. Presença do acompanhante permitida & 25 & Sim & 100 \\
\hline \multicolumn{4}{|l|}{ Momento antes da expulsão (ou antes da cesariana) } \\
\hline 9. Identificação do profissional responsável & 22 & $\operatorname{Sim}$ & 88 \\
\hline \multirow[t]{2}{*}{ 10. Indicação para administração de antibiótico à gestante } & 19 & Infecções & 76 \\
\hline & 14 & Profilaxia cirúrgica & 56 \\
\hline \multirow[t]{2}{*}{ 11. Indicação para administração de Sulfato de Magnésio } & 24 & Síndrome Hipertensiva & 96 \\
\hline & 5 & Convulsão & 20 \\
\hline 12. Indicação para administração de Antihipertensivos & 20 & Crises hipertensivas & 80 \\
\hline $\begin{array}{l}\text { 13. Disponibilidade de materiais higiene e luvas para realização } \\
\text { do parto }\end{array}$ & 25 & $\operatorname{Sim}$ & 100 \\
\hline 14. Disponibilidade de Ocitocina & 24 & Sim & 96 \\
\hline Administração de Ocitocina após o parto & 25 & Imediatamente & 100 \\
\hline 15. Cuidados realizados na mãe após a dequitação/expulsão da & 21 & Revisão do trajeto & 84 \\
\hline \multirow[t]{2}{*}{ placenta } & 14 & Suturas & 56 \\
\hline & 08 & Sinais Vitais & 32 \\
\hline \multicolumn{4}{|l|}{ Logo após o parto } \\
\hline 16. Conduta se há hemorragia & 19 & Massagem uterina & 76 \\
\hline
\end{tabular}




\begin{tabular}{llll} 
& 18 Medicação & 72 \\
17. Indicação para administração de antibióticos & 13 Comunicar Equipe & 52 \\
& 22 Infecções & 88 \\
18. Indicação para administração de sulfato de magnésio & 07 Febre & 28 \\
19. Indicação para administração de anti-hipertensivo & 24 HA Gestacional & 20 \\
\hline
\end{tabular}

Legenda: BCFs: Batimentos Cardíacos Fetais; min: minutos; FC: Frequência Cardíaca; TAX: Temperatura Axilar; PA: Pressão Arterial; HA: Hipertensão Arterial; AVP: Acesso Venoso Periférico.

Apresentam-se, na Tabela 2, os itens verificados no segundo momento da lista por 41 profissionais, sendo 16 enfermeiras da UCO, nove médicos obstetras e 16 médicos da neonatologia. As práticas realizadas foram a verificação da disponibilidade de materiais para o recém-nascido, o início da amamentação e o contato pele a pele.

Tabela 2 - Itens da LVPS realizados por médicos obstetras, neonatologistas e enfermeiras na UCO. Porto Alegre - RS, 2019.

\begin{tabular}{|c|c|c|c|}
\hline \multirow{2}{*}{$\begin{array}{l}\text { Variável } \\
\text { Momento antes da expulsão (ou antes da cesariana) }\end{array}$} & \multicolumn{2}{|c|}{$\mathrm{n}=\mathbf{4 1}$} & \multirow[t]{2}{*}{$\%$} \\
\hline & & & \\
\hline 20. Disponibilidade de compressas limpas para o RN & 41 & Sim & 100 \\
\hline $\begin{array}{l}\text { 21. Disponibilidade de tesoura estéril para cortar o cordão } \\
\text { umbilical }\end{array}$ & 41 & $\operatorname{Sim}$ & 100 \\
\hline 22. Início da amamentação & 41 & Sim & 100 \\
\hline 23. Contato pele à pele & 41 & Sim & 100 \\
\hline 24. Orientações à mulher e ao acompanhante sobre sinais e & 15 & Amamentação & 36,6 \\
\hline \multirow[t]{4}{*}{ perigos para solicitar auxílio após o parto } & 09 & Dor & 22,0 \\
\hline & 08 & Sangramento & 19,5 \\
\hline & 05 & Alimentação & 12,2 \\
\hline & 05 & Eliminações & 12,2 \\
\hline
\end{tabular}

Legenda: RN: Recém-nascido.

Identificou-se, na Tabela 3, referente ao segundo e ao terceiro momento da lista, as ações realizadas por 32 profissionais (16 enfermeiros da UCO e 16 médicos neonatologistas), relacionadas aos cuidados ao recém-nascido. Destes, a disponibilidade de materiais e o cuidado de secagem, são práticas realizadas. No entanto, as práticas como cuidados especiais e uso de antibióticos não são realizadas conforme preconizado pela LVPS. 
9 | Santos MC, Pedroni VS, Carlotto FD, Silva SC, Gouveia HG, Vieira LB

Tabela 3 - Itens da LVPS realizados por médicos neonatologistas e enfermeiras na UCO. Porto Alegre - RS, 2019.

\begin{tabular}{llll}
\hline Variável & $\mathbf{n}=\mathbf{3 2}$ & $\mathbf{\%}$ \\
\hline Momento antes da expulsão (ou cesariana) & & & \\
25. Disponibilidade de dispositivo de aspiração & 32 & Sim & 100 \\
26. Disponibilidade de balão e máscara & 32 & Sim & 100 \\
27. Cuidados realizados com RN logo após o & 22 & Secar & 68,8 \\
nascimento & 13 & Clamp & 40,6 \\
& 13 & Exame físico & 40,6 \\
& 11 & Temperatura & 34,4 \\
& 11 & Pesar & 34,4 \\
& 09 & Identificação & 28,1 \\
28. Necessidade de cuidados especiais ao RN & 27 & FR alterada & 84,4 \\
& 21 & Saturação de O2 & 65,6 \\
& & inferior a 90\% & \\
& 16 & Hipoglicemia & 50 \\
& 14 & Prematuridade & 43,8 \\
& 12 & Alteração FC & 37,5 \\
& 08 & Malformações & 25 \\
& 08 & Alteração Tax & 25 \\
& 05 & Infecção & 15,6 \\
29. Indicação para administração de antibióticos & 15 & Infecção RN & 46,9 \\
ao RN & 09 & Infecção materna & 28,1 \\
& 02 & Febre & 6,3 \\
\hline
\end{tabular}

Legenda: RN: Recém-nascido; FC: Frequência Cardíaca; TAX: Temperatura Axilar.

Por conseguinte, nas Tabelas 4 e 5, descrevem-se as ações realizadas na UIO no momento antes da alta, sendo respondidas por 19 e 26 profissionais respectivamente. Evidenciou-se que o uso de medicações, situações de adiamento da alta hospitalar e conduta quando há sangramento anormal são práticas realizadas pelos profissionais. 
Tabela 4 - Itens da LVPS realizados por médicos obstetras e enfermeiras na UIO. Porto Alegre - RS, 2019.

\begin{tabular}{|c|c|c|c|}
\hline Variável & \multicolumn{2}{|c|}{$\mathbf{n}=\mathbf{1 9}$} & $\%$ \\
\hline \multicolumn{4}{|l|}{ Antes da Alta } \\
\hline 30. Indicação para administração de antibióticos para & 13 & Infecções & 68,4 \\
\hline gestante & 02 & Procedimentos cirúrgicos & 10,5 \\
\hline \multirow[t]{2}{*}{ 31. Indicação para administração de Sulfato de Magnésio } & 19 & Síndrome Hipertensiva & 36,8 \\
\hline & 05 & Não realizado na UIO & 26,3 \\
\hline \multirow[t]{2}{*}{ 32. Indicação para administração de Anti-hipertensivos } & 19 & Crises Hipertensivas & 57,9 \\
\hline & 07 & HA Crônica & 36,8 \\
\hline \multirow[t]{3}{*}{ 33. Situações clínicas que adiam a alta hospitalar da puérpera } & 06 & Sangramento & 31,6 \\
\hline & 06 & Infecção & 31,6 \\
\hline & 04 & RN sem alta & 21,1 \\
\hline \multirow[t]{4}{*}{ 34. Presença de hemorragia antes da alta } & 09 & Comunicar equipe médica & 47,4 \\
\hline & 08 & Acesso Venoso & 42,1 \\
\hline & 05 & Massagem Uterina & 26,3 \\
\hline & 02 & Monitorização & 10,5 \\
\hline 35. Marcada consulta para puérpera & 10 & Sim & 52,6 \\
\hline 36. Orientação sobre planejamento reprodutivo & 12 & $\operatorname{Sim}$ & 63,2 \\
\hline \multirow[t]{3}{*}{ 37. Orientação para solicitar auxílio após a alta hospitalar } & 05 & Sangramento & 26,3 \\
\hline & 04 & Dor & 21.0 \\
\hline & 07 & Alterações na FO & 36,8 \\
\hline
\end{tabular}

Legenda: RN: Recém-nascido; UIO: Unidade de Internação Obstétrica; HÁ: Hipertensão Arterial; FO: Ferida Operatória.

Tabela 5 - Itens da LVPS realizados por médicos neonatologistas e enfermeiras da UIO. Porto Alegre - RS, 2019.

\begin{tabular}{|c|c|c|c|}
\hline Variável & \multicolumn{2}{|c|}{$\mathrm{n}=\mathbf{2 6}$} & $\%$ \\
\hline \multicolumn{4}{|l|}{ Antes da Alta } \\
\hline \multirow[t]{4}{*}{ 38. Indicação para administração de antibióticos ao RN } & 09 & Mãe com Sífilis & 34,6 \\
\hline & 04 & Infecção & 15,4 \\
\hline & 03 & Profilaxia para disfunção renal & 11,5 \\
\hline & 02 & Hemograma infeccioso & 07,7 \\
\hline 39. Alta suspensa se dificuldade em amamentação & 23 & Sim & 88,5 \\
\hline 40. Marcação de consulta ao RN & 18 & Sim & 69,2 \\
\hline \multirow{4}{*}{$\begin{array}{l}\text { 41. Orientação para solicitar auxílio apó } \\
\text { hospitalar }\end{array}$} & 14 & RN não mama & 53,8 \\
\hline & & & \\
\hline & 03 & Alterações do Coto umbilical & \\
\hline & 03 & Alterações do Sensório & 11,5 \\
\hline \multirow{3}{*}{$\begin{array}{l}\text { 42. Outras práticas relevantes para a assistência e que } \\
\text { envolvem a segurança do paciente }\end{array}$} & 12 & RN mantido no berço & 46,1 \\
\hline & 11 & Prevenção de quedas & 42,3 \\
\hline & 10 & Pulseira de identificação & 38,4 \\
\hline
\end{tabular}

Legenda: RN: Recém-nascido. 
11 | Santos MC, Pedroni VS, Carlotto FD, Silva SC, Gouveia HG, Vieira LB

Algumas orientações sobre situações de riscos como alteração da pressão arterial materna, indicação para administração de antibióticos ao recém-nascido e marcação de consultas de seguimento não são realizadas conforme preconizado pela LVPS. Indicando a necessidade de melhorias para garantir a implementação dessas práticas.

\section{Discussão}

A LVPS da OMS foi desenvolvida para auxiliar os profissionais de saúde a desempenharem práticas essenciais voltadas à segurança da mulher e ao recém-nascido no trabalho de parto, parto e puerpério. Para sua elaboração, considerou-se a sequência de rotina dos eventos desde a admissão até a alta hospitalar, sendo as práticas essenciais agrupadas em quatro momentos cruciais da assistência ao parto e ao nascimento, promovendo-se boas práticas e prevenindo-se as principais causas da mortalidade materna e neonatal. ${ }^{4}$

Quando a gestante ingressa no ambiente hospitalar é realizado seu acolhimento pela equipe de saúde da UCO e a verificação da disponibilidade de recursos para o atendimento integral (72\%), favorecendo-se o acesso aos serviços de saúde, que é um pilar para a prevenção da mortalidade perinatal juntamente com a qualificação da assistência oferecida. ${ }^{11}$ Também é verificado se a gestante tem alergia (96\%), caso seja necessária a utilização de fármacos. Deste modo, desde sua inserção ao ambiente hospitalar, essas condutas têm sido preconizadas, favorecendo a segurança e a assistência necessárias às mulheres, prevenindo-se eventos adversos a medicações. ${ }^{13-14}$

Outra ferramenta importante é o partograma, prática essencial e amplamente utilizada pela equipe de saúde da instituição do estudo (96\%). O partograma auxilia os profissionais de saúde no monitoramento do progresso do parto, evitando trabalhos de parto prolongados e procedimentos operatórios desnecessários, reduzindo-se a mortalidade neonatal. ${ }^{15}$ 
A periodicidade dos registros das contrações uterinas, os batimentos cardiofetais e a frequência cardíaca materna no partograma, de acordo com a recomendação da OMS, devem ser verificados a cada 30 minutos. ${ }^{4}$ Assim, conforme indicado, considera-se que há oportunidade de melhoria quanto à frequência da realização dessas práticas, visto que os registros das contrações uterinas $(48 \%)$ e batimentos cardiofetais (56\%) são realizados a cada 30 minutos, e sendo que a frequência cardíaca materna é realizada a cada seis horas (80\%), uma vez que não houve padronização quanto à frequência de realização dessas avaliações. Em relação à mensuração da temperatura corporal, a indicação é que seja registrada a cada duas horas, e a tensão arterial sistêmica a cada quatro horas; ${ }^{4}$ porém, percebe-se que os profissionais realizam esta aferição a cada seis horas $(76 \%)$.

Em relação ao uso de medicações, a LVPS prevê analisar a utilização de antibióticos, antihipertensivos e sulfato de magnésio indicados respectivamente para sinais clínicos de infecções, hipertensão e pré-eclâmpsia. Estas alterações fisiológicas encontram-se entre as principais causas de morte materna no Brasil. ${ }^{16} \mathrm{O}$ uso de antibiótico é indicado na presença de sinais clínicos de infecção, como febre, corrimento vaginal fétido e bolsa rota maior que 18 horas e a administração deve ocorrer o mais rapidamente possível. ${ }^{4}$ Essa prática assistencial na instituição investigada está em conformidade, pois $92 \%$ dos profissionais indicaram seu uso para situações de infecção e $60 \%$ em situações de bolsa rota.

Ainda, relacionado à utilização de fármacos, destaca-se diretriz internacional, a qual recomenda a administração de sulfato de magnésio se pressão arterial diastólica for $\geq 110 \mathrm{mmHg}$ e proteinúria maior que três ou pressão arterial diastólica for $\geq 90 \mathrm{mmHg}$, proteinúria for maior que dois e haja sinais clínicos de hipertensão, como cefaleia e distúrbio visual. ${ }^{4}$ Estudo demonstra a efetividade deste fármaco em comparação com outros tratamentos para hipertensão, sendo também utilizado para neuroproteção em prematuros. ${ }^{17}$ 
13 | Santos MC, Pedroni VS, Carlotto FD, Silva SC, Gouveia HG, Vieira LB

Os achados do presente estudo também apontaram a indicação desse fármaco para os casos de prematuridade, visto que seu efeito bloqueador do receptor N-metil D-Aspartato (NMDA) lhe confere-lhe características analgésicas e sedativa. ${ }^{18}$ Quanto à prescrição de anti-hipertensivos, foi indicada para as síndromes hipertensivas (88\%) e hipertensão arterial crônica (52\%), o que merece um alerta, visto que não foi relatado por todos os profissionais e são drogas importantes para controle dos níveis pressóricos e prevenção de agravamentos tanto para mulher quanto para o feto. ${ }^{4}$ Foi relatado por um número expressivo de profissionais a utilização desses fármacos conforme preconizados pela OMS, todavia algumas situações, como convulsão (20\%) foram pouco relatadas; e a realização de exames, como a proteinúria, não foram citados pelos profissionais.

Além dos cuidados com a mãe, também deve-se atentar ao recém-nascido nas primeiras horas de vida. Caso necessite receber antibiótico, esse é prescrito pela equipe médica, considerando-se o histórico materno e os achados clínicos, tais como alteração da frequência respiratória, retração torácica, gemidos, hipotermia, febre e reação lenta aos estímulos. ${ }^{4}$ Caso o recém-nascido necessite de antibióticos, ele é transferido para a unidade de neonatologia para ser monitorado.

A conferência dos materiais necessários para a realização do parto é feita pela equipe de enfermagem, o que é fundamental para garantir a segurança da paciente. O preparo antecipado de ocitocina é recomendado, o que permite que o profissional de saúde possa realizar manobras de reanimação ao recém-nascido, visto que devem iniciar-se no primeiro minuto de vida. ${ }^{19}$ Os achados do estudo relacionados a essas práticas estão em conformidade sugerido pela LVPS, visto que é feita a conferência $(100 \%)$ de todos os materiais necessários para a realização do parto e atendimento à puérpera e ao recém-nascido.

Outra prática de conferência refere-se à lavagem de mãos por parte dos profissionais de saúde, que podem conter agentes transmissores de infecções às gestantes. ${ }^{20}$ Desse modo, 
disponibilizar recursos materiais para realização dos exames vaginais e para higiene das mãos são necessários para à assistência segura estando esta prática essencial de acordo com a LVPS.

Referente às práticas realizadas com a mãe após o nascimento, primeiramente, o profissional obstetra deve verificar que não tenham mais fetos para nascer, logo em seguida é recomendada a administração da ocitocina no primeiro minuto, clampeamento do cordão umbilical entre o 1-3 minutos de vida do recém-nascido e massagem do útero após a expulsão da placenta, técnica que assegura que o útero esteja contraído, evitando-se a hemorragia pósparto. ${ }^{4}$ Os profissionais relatam a revisão do trajeto, suturas, verificação dos sinais vitais, higiene e limpeza e verificação do globo de segurança, podendo-se acrescentar alguns itens e torná-los padrão, conforme indicado pela LVPS.

Caso haja sangramento anormal, iniciam-se as massagens uterinas, medicações, e a equipe médica é comunicada, quando não está presente, são realizados o monitoramento e a infusão de soros e volumes. A Lista adiciona práticas, como manter a mãe aquecida. ${ }^{4}$ Ressalta-se que a hemorragia pós-parto é a segunda principal causa de morte materna no Brasil ${ }^{16}$ e pode acontecer por diversas situações, sendo necessária a atenção da equipe e a realização de intervenções segundo os sinais clínicos da mulher. Devido à importância dessa situação, é fundamental a aplicação de protocolos institucionais que organizem a assistência da equipe multiprofissional.

Dentre os cuidados realizados com o recém-nascido, incluem-se primeiramente a secagem para se manter temperatura corporal adequada; com esse processo, o recém-nascido já é estimulado para chorar e respirar. O cordão deve ser laqueado e cortado com tesoura estéril para se prevenirem infecções. ${ }^{4}$ Caso o recém-nascido não respire imediatamente após o nascimento, deve ser utilizado o dispositivo de aspiração, máscara e todo o suporte necessário para reanimá-lo.

Nas situações em que o recém-nascido apresenta boas condições de vitalidade, estimulase o contato pele à pele e inicia-se o aleitamento materno. Destaca-se que a recomendação para o sucesso do aleitamento materno, sugerido pela Iniciativa Hospital Amigo da Criança, é o 
15 | Santos MC, Pedroni VS, Carlotto FD, Silva SC, Gouveia HG, Vieira LB

contato pele à pele por uma hora. Trata-se de um momento importante para o estímulo e incentivo à amamentação. ${ }^{21}$ Desse modo, considera-se que essas ações estão conforme o preconizado, pois $96,9 \%$ dos profissionais proporcionam o contato pele à pele e incentivam a amamentação quando possível. A LVPS tem demonstrado ser um instrumento que mantém essas práticas, fortalecendo a cultura e a segurança do paciente. ${ }^{7}$

A OMS recomenda que no momento imediato anterior à expulsão (ou antes da cesárea), caracterizado por ser um momento ápice para possíveis complicações à gestante e ao recémnascido, a equipe de saúde certifique-se de que o assistente responsável pelo parto seja identificado, visto que, na presença de complicações durante a assistência desse momento, esse profissional poderá ser acionado. No protocolo assistencial da instituição investigada, essa prática está em conformidade ao preconizado, ou seja, dispõe-se de recurso humano para este fim. ${ }^{4}$

A presença do acompanhante é garantida por lei desde 2011, sendo uma prática essencial dos profissionais de saúde, por estimular diversos benefícios à gestante e ao recém-nascido, como maior tranquilidade à mulher, menor uso de analgésicos, favorecimento da evolução do parto normal e fortalecimento entre os vínculo familiares. ${ }^{4}$ Essa prática contribui para a segurança do binômio mãe-bebê e configura-se como um importante suporte contínuo durante o trabalho de parto e parto, estendendo-se os cuidados no pós-parto imediato e no alojamento conjunto. ${ }^{22}$

A participação positiva do acompanhante, o suporte físico e emocional que fornece condiz com a humanização deste processo. Além disso, estudo relaciona sua importância com a diminuição da dor nesse momento. ${ }^{22}$ As orientações realizadas pela equipe de saúde fornecem subsídios à mulher e a quem a acompanha, propiciando conforto e calma para que eles possam identificar alterações clínicas que necessitem de ajuda. Essas orientações são oportunidades para que equipe de saúde forneça um atendimento holístico e integral. ${ }^{22}$

O profissional de saúde deve orientar a mulher e o acompanhante a solicitar auxílio imediato, caso ocorra algum sinal de perigo, como distúrbio visual, cefaleia, dor abdominal, 
hemorragia, dificuldade em urinar ou respirar e nos casos em que o bebê apresente dificuldades de amamentação, esforço para respirar e não esteja reativo aos estímulos. ${ }^{23}$ Essa ação de orientação não foi adequada aos parâmetros da OMS, uma vez que as orientações elencadas pelos profissionais não contemplaram todos os sinais de perigo que merecem atenção nesse momento da LVPS.

$\mathrm{Na}$ ocasião da alta, também são dadas orientações sobre o planejamento reprodutivo e sobre a abordagem dos diferentes tipos e indicações de métodos contraceptivos (considerandose os critérios de elegibilidade), de modo que possibilitem à mulher optar pelo método que melhor se adeque ao seu contexto de vida e social. A educação em saúde é fundamental para que haja a continuidade nos cuidados que serão realizados com a mulher e o recém-nascido, sendo importante a referência à rede de atenção básica à saúde. ${ }^{4}$ Enfermeiras relatam que tais orientações e o agendamento para seguimento na atenção básica no município são realizados devido à comunicação com a rede municipal prevista pelo SUS. Todavia, estudo piloto na Índia destaca a falta dos encaminhamentos e dessa rede interligada. ${ }^{7}$

A alta é indicada quando se verifica que a puérpera e o recém-nascido estão bem e conseguirão suprir suas necessidades fora do ambiente hospitalar. Caso o recém-nascido não esteja se alimentando, a mesma deve ser suspensa. ${ }^{4}$ Em sua maioria, os profissionais relatam que para os recém-nascidos que não estão com a amamentação estabelecida ou o façam com muita dificuldade a permanência no hospital é mantida, para que seja fortalecido o aleitamento materno, o que corrobora com as recomendações internacionais da OMS.

Outras práticas relatadas pelos profissionais, que reforçam a segurança do paciente, são: manter-se o recém-nascido no berço e utilizar-se pulseira de identificação. Essas práticas são condizentes com os protocolos e as recomendações internacionais a respeito da segurança do paciente e devem ser adicionadas à LVPS. Manter-se o recém-nascido no berço está relacionado 
17 | Santos MC, Pedroni VS, Carlotto FD, Silva SC, Gouveia HG, Vieira LB

à prevenção do risco de quedas e as pesquisas sugerem que isso esteja contemplado nos protocolos das instituições e nas prescrições de enfermagem. ${ }^{24}$

Estudo $^{25}$ que avaliou a implementação da LVPS em vários países salienta a necessidade de participação dos profissionais e gestores dos serviços de saúde para a adaptação e aceitação. Visando a sua correta utilização como instrumento que favoreça à manutenção das práticas já realizadas na instituição e à implementação de novas para a segurança do binômio mãe-bebê.

\section{Conclusão}

O uso da LVPS pode ser útil para se manterem as ações já realizadas, que são preconizadas, e para a inclusão de novos itens recomendados pela OMS, de modo a potencializá-las, favorecendo-se a segurança do paciente com a padronização de condutas no processo assistencial ao binômio mãe-bebê. As ações como: indicação para o início do partograma, uso de medicações, verificação se a gestante tem alergia, cuidados com o recémnascido, início da amamentação, incentivo da participação do acompanhante, administração de ocitocina, disponibilidade de materiais estéreis para consulta e parto, disponibilidade de materiais para lavagem de mãos e aleitamento materno são práticas essenciais da LVPS da OMS. No presente estudo, verificou-se que as mesmas já estão implementadas no processo de trabalho da equipe de saúde da área obstétrica do Hospital Universitário em questão.

Concluiu-se que a LVPS é uma ferramenta inovadora na assistência obstétrica, e que sua implementação acrescenta oportunidade de melhorias e qualificação às práticas essenciais, que estão parcialmente sendo realizadas na instituição estudada, com destaque para as orientações sobre os sinais clínicos de agravamento da condição de saúde e a periodicidade de mensuração e registro de parâmetros no partograma. Para que sejam assegurados a manutenção e o aprimoramento das práticas essenciais, com vistas à qualificação da assistência obstétrica, 
recomenda-se o uso de estratégias e tecnologias que fortaleçam a cultura de segurança do paciente na instituição.

Como limitações do estudo, foram incluídos profissionais médicos e enfermeiros, todavia, técnicos de enfermagem e estudantes residentes exercem participação ativa no cuidado às gestantes e ao recém-nascido e sua inclusão em estudos posteriores poderiam fortalecer a segurança do paciente nesses locais de saúde.

\section{Referências}

1. Marcolin AC. Qualidade e segurança: caminhos para o sucesso do redesenho do modelo de cuidado obstétrico. Rev Bras Ginecol Obstet. 2015;37(10):441-5. doi: 10.1590/SO100-720320150005472

2. Organización Mundial de la Salud. Mortalidad materna [Internet]. 2015 [acceso en 2019 mayo 05]. Disponible en: http://www.who.int/mediacentre/factsheets/fs348/es/

3. Mason E, McDougall L, Lawn JE, Gupta A, Claeson M, Pillay Y, et al. From evidence to action to deliver a healthy start for the next generation. Lancet. 2014;384(9941):455-67. doi: 10.1016/S01406736(14)60750-9

4. Organização Mundial da Saúde. Guia de implementação da Lista de Verificação da OMS para partos seguros: melhorar a qualidade dos partos realizados em unidades de saúde para as mães e os recémnascidos [Internet]. Genebra: Organização Mundial da Saúde; 2017 [acesso em 2019 abr 20]. Disponível em: http://apps.who.int/iris/bitstream/10665/199177/5/9789248549458-por.pdf?ua=1

5. Kabongo L, Gass J, Kivondo B, Kara N, Semrau K, Hirschhorn LR. Implementing the WHO Safe Childbirth Checklist: lessons learnt on a quality improvement initiative to improve mother and newborn care at Gobabis District Hospital, Namibia. BMJ Open Qual. 2017;6(2). doi: 10.1136/bmjoq-2017-000145

6. Semrau EAK, Hirschhorn LR, Delaney MM, Singh VP, Saurastri R, Sharma N, et al. Outcomes of a coaching-based WHO Safe Childbirth Checklist Program in India. N Engl J Med. 2017;377(24):2387-8. doi: 10.1056/NEJMoa1701075

7. Spector MJ, Agrawal P, Kodkany B, Lipsitz S, Lashoher A, Dziekan G, et al. Improving quality of care for maternal and newborn health: prospective pilot study of the WHO Safe Childbirth Checklist Program. PLoS One. 2012;7(5). doi: 10.1371/journal.pone.0035151

8. Semrau KEA, Hirschhorn LR, Kodkany B, Spector JM, Tuller DE, King G, et al. Effectiveness of the WHO Safe Childbirth Checklist program in reducing severe maternal, fetal, and newborn harm in Uttar Pradesh, India: study protocol for a matched-pair, cluster-randomized controlled trial. Trials. 
19 | Santos MC, Pedroni VS, Carlotto FD, Silva SC, Gouveia HG, Vieira LB

2016;17:576. doi: https://doi.org/10.1186/s13063-016-1673-x

9. Kumar S, Yadav V, Balasubramaniam S, Jain Y, Shekhar C, Saran K, et al. Effectiveness of the WHO SCC on improving adherence to essential practices during childbirth, in resource constrained settings. BMC Pregnancy Childbirth. 2016;16(345). doi:10.1186/s12884-016-1139-x

10. Carvalho ICBM, Rosendo TMSS, Freitas MR, Silva EMM, Medeiros WR, Moutinho NF, et al. Adaptação e validação da Lista de Verificação do Parto Seguro da Organização Mundial da Saúde para o contexto brasileiro. Rev Bras Saúde Mater Infant [Online]. 2018;18(2):401-18. doi: 10.1590/180693042018000200009

11. Koblinsky M, Moyer CA, Calvert C, Campbell J, Campbell OM, Feigl AB, et al. Quality maternity care for every woman, everywhere: a call to action. Lancet. 2016;388(10057):2307-20. doi: 10.1016/S01406736(16)31333-2

12. Praxedes AO, Arrais L, Araújo MAA, Silva EMM, Gama ZAS, Freitas MR. Avaliação da adesão à Lista de Verificação de Segurança no Parto em uma maternidade pública no Nordeste do Brasil. Cad Saúde Pública [Online]. 2017;33(10):e00034516. doi: 10.1590/0102-311x00034516

13. Ministério da Saúde (BR), Secretaria de Atenção à Saúde, Departamento de Ações Programáticas Estratégicas, Coordenação Geral de Saúde das Mulheres - DAPES/SAS/MS. ApiceOn - Aprimoramento e inovação no cuidado e ensino em obstetrícia e neonatologia [Internet]. 2017 [acesso em 2019 maio 18]. Disponível em: http://portalarquivos.saude.gov.br/images/pdf/2017/agosto/18/Apice-On-2017-08-11.pdf

14. Giannattasio MB, Taniguchi FP. Avaliação da segurança do paciente em cirurgia cardíaca de um hospital público. Rev SOBECC. 2016;21(3);125-31. doi:10.5327/Z1414-4425201600030002

15. World Health Organization partograph in management of labour. World Health Organization Maternal Health and Safe Motherhood Programme. Lancet [Internet]. 1994 [cited 2019 Jul 08]; 343(8910):1404-399. Available from: https://www.ncbi.nlm.nih.gov/pubmed/7910888

16. Dias JMG, Oliveira APS, Cipolotti R, Monteiro BKSM, Pereira RO. Mortalidade materna. Rev Méd Minas Gerais [Internet]. 2015 Apr [cited 2019 Aug 15];25(2):168-74. Available from: http://www.rmmg.org/artigo/detalhes/1771

17. Rodríguez-Hernandéz PA, Beltrán-Avendaño MA. Aproximación a la farmacología del sulfato de magnesio desde la perspectiva obstétrica. MedUNAB. 2016;19(1):25-32. doi:10.29375/01237047.2327

18. Pinheiro ACB, Santos ALT, Campos AN, Duarte LM, Oliveira MBG, Silva SAB, et al. Efeito neuroprotetor do sulfato de magnésio em recém-nascidos abaixo de 32 semanas admitidos em Unidade de Terapia Intensiva Neonatal. Rev Méd Minas Gerais [Internet]. 2018 [acesso em 2019 set 20];28(4):63-71. Disponível em: http://www.rmmg.org/artigo/detalhes/2309

19. Schardosim JM, Rodrigues NLA, Rattner D. Parâmetros utilizados na avaliação do bem-estar 
do bebê no nascimento. Av Enferm. 2018;36(2):197-208. doi: 10.15446/av.enferm.v36n2.67809

20. World Health Organization (WHO). Pregnancy, childbirth, postpartum and newborn Care: a guide for essential practice. 3rd. ed. [Internet]. 2015 [cited 2019 Sept 18]; Available from: https:/www.who.int/maternal_child_adolescent/documents/imca-essential-practice-guide/en/

21. Silva CM, Pereira SCL, Passos IR, Santos LC. Fatores associados ao contato pele a pele entre mãe/filho e amamentação na sala de parto. Rev Nutr (Online). 2016;29(4):457-71. doi: 10.1590/1678-98652016000400002

22. Santos ALS, Oliveira ARS, Amorim T, Silva UL. O acompanhante no trabalho de parto sob a perspectiva da puérpera. Rev Enferm UFSM. 2015;5(3):531-40. doi: 10.5902/2179769217337

23. Colli M, Zani AV. Validação de um plano de alta de enfermagem para gestantes e puérperas de alto risco. REME Rev Min Enferm. 2016;20(e934):1-7. doi: 10.5935/1415-2762.20160004

24. Silva RSS, Rocha SS, Gouveia MTO, Dantas ALB, Santos JDM, Carvalho NAR. Wearing identification wristbands: implications for newborn safety in maternity hospitals. Esc Anna Nery Rev Enferm. 2019;23(2):e20180222. doi: 10.1590/2177-9465-EAN-2018-0222

25 Perry WRG, Nejad SB, Tuomisto K, Kara N, Ross N, Dilip TR, et al. Implementing the WHO Safe Childbirth Checklist: lessons from a global collaboration. BMJ Glob Health. 2017;2:e000241. doi: 10.1136/bmjgh-2016-000241

Editor Científico Chefe: Cristiane Cardoso de Paula

\section{Autor correspondente}

Letícia Becker Vieira

E-mail: lebvieira@hotmail.com

Endreço: R. São Manoel, 963 - Rio Branco, Porto Alegre - RS, 90620-110

\section{Contribuições de Autoria}

1 - Maxuel Cruz dos Santos

Cconcepção do estudo, coleta de dados, análise e interpretação dos dados, discussão dos resultados, redação e/ou revisão crítica do conteúdo,

\section{2 - Vitoria Sandri Pedroni}

Contribuições: coleta de dados, redação e/ou revisão crítica do conteúdo

3 - Franciela Delazeri Carlotto 
21 Santos MC, Pedroni VS, Carlotto FD, Silva SC, Gouveia HG, Vieira LB

Contribuições: coleta de dados, redação e/ou revisão crítica do conteúdo

\section{4 - Silvana Cruz da Silva}

Contribuições: análise e interpretação dos dados, discussão dos resultados, redação e/ou revisão crítica do conteúdo, revisão e aprovação da versão final

\section{5 - Helga Geremias Gouveia}

Contribuições: concepção do estudo, análise e interpretação dos dados, discussão dos resultados, redação e/ou revisão crítica do conteúdo, revisão e aprovação da versão final

\section{6 - Letícia Becker Vieira}

Concepção do estudo, análise e interpretação dos dados, discussão dos resultados, redação e/ou revisão crítica do conteúdo, revisão e aprovação da versão final

\section{Como citar este artigo}

Santos MC, Pedroni VS, Carlotto FD, Silva SC, Gouveia HG, Vieira LB. Prática segura para partos em hospital universitário. Rev. Enferm. UFSM. 2020 [Acesso em: Anos Mês Dia]; vol.10 e80: 1-21. DOI:https://doi.org/10.5902/2179769241489 